\section{Protocol that shortens hospital stay after laparoscopic donor nephrectomy}

New research from the University of California, Los Angeles, reports that a combination protocol of ketorolac analgesia and strict bowel preparation reduced the time in hospital following laparoscopic live-donor nephrectomy.

All patients who underwent laparoscopic donor nephrectomy $(n=300)$ between 2000 and 2005 at the authors' institution were put on 2 days of clear liquid diet before surgery, and instructed to take two bottles of magnesium citrate 1 day before surgery, to self-administer a Fleets enema the evening before surgery, and to fast from midnight. After surgery, $30 \mathrm{mg}$ of ketorolac-an NSAID for the control of postoperative pain-was administered intravenously every 6 hours for 48 hours, with additional narcotics given when needed.

All patients tolerated the preoperative regimen. The mean operative time was $180 \pm 55 \mathrm{~min}$, mean blood loss was $80 \pm 50 \mathrm{ml}$, and warm ischemia time was $4 \pm 2 \mathrm{~min}$. The mean hospital stay was 1.1 days (range $1-3$ ), with $96.7 \%$ of patients able to leave on postoperative day 1 . There were no readmissions or emergency room visits. On the day after surgery, over $97 \%$ of patients could eat a normal lunch and walk with oral pain medication. Patients requiring longer hospital stays of 2-3 days required intravenous analgesia $(n=6)$ or had postoperative ileus $(n=4)$. No renal impairment was reported.

The authors attribute the reduced hospital stay to better analgesia and a faster return to normal bowel function, resulting from the bowel preparation regimen, but note that this was not a controlled randomized study.

Original article Breda A et al. (2007) Association of bowel rest and ketorolac analgesia with short hospital stay after laparoscopic donor nephrectomy. Urology 69: 828-831

\section{Postpuberty corrective surgery doubles risk of testicular cancer in cryptorchidism}

Surgical treatment for cryptorchidism before puberty decreases the risk of testicular cancer, according to a large Swedish cohort study.

Cryptorchidism is a known risk factor for testicular cancer, but the relationship between age at treatment and testicular cancer risk is controversial. To address this issue, Pettersson and colleagues from the Karolinska Institutet in Stockholm reviewed the records of almost 17,000 Swedish men who underwent surgery to correct cryptorchidism before the age of 20 years. Patients were followed for a total of 209,984 person-years, with follow-up starting 1 year after surgery to reduce the risk of including patients who already had testicular cancer. A nationwide cancer registry was used to monitor the occurrence of testicular cancer in the cohort, ensuring complete follow-up. The relative risk of testicular cancer in the cohort was estimated and compared with that in the general population.

In total, 56 patients in the cohort developed testicular cancer during the follow-up period. Patients who underwent surgery before the age of 13 years had a relative risk of 2.23 (95\% Cl 1.58-3.06) of developing testicular cancer compared with the Swedish general population, whereas those who underwent surgery after the age of 13 years had a relative risk of 5.40 (95\% Cl, 3.20-8.53).

Although the risk of testicular cancer is thought to be determined before birth, these findings suggest that puberty may be another important event in testicular carcinogenesis. This study supports the current recommendation that patients with cryptorchidism are treated early.

Original article Pettersson A et al. (2007) Age at surgery for undescended testis and risk of testicular cancer. $N$ Engl J Med 356: $1835-1841$

\section{Fertility concerns over sildenafil citrate use}

A recent study from the UK suggests that sildenafil citrate might adversely affect male fertility. Sildenafil is increasingly being used recreationally by younger men who want to start families, and assisted conception units are increasingly prescribing sildenafil to patients to help them to provide a semen sample on demand. It is worrying, therefore, that this research has suggested that sildenafil might affect sperm function.

The authors of this study used computerassisted semen analysis and acrosome reaction testing by fluorescein-isothiocyanate-labeled peanut agglutinin staining to analyze the motility of sperm from 57 men after exposure to 